\title{
Orally delivered sour cherry seed extract (SCSE) affects cardiovascular and hematological parameters in humans
}

Original article

Zoltan Csiki ${ }^{* 1}$, Agnes Papp-Bata ${ }^{* 2}$, Attila Czompa ${ }^{3}$, Aniko Nagy ${ }^{1,4}$, Istvan Bak ${ }^{3}$, Istvan Lekli ${ }^{3}$, Andras Javor ${ }^{4}$, David D. Haines ${ }^{3}$, Gyorgy Balla ${ }^{5}$, Arpad Tosaki ${ }^{3}$

* The first two authors contributed equally to this work

Running head: toxicological evaluation of sour cherry seed extract

${ }^{1}$ Division of Clinical Immunology, Institute of Medicine, Faculty of Medicine, University of Debrecen, Debrecen, Hungary

${ }^{2}$ Faculty of Applied Economics and Rural Development, University of Debrecen, Debrecen, Hungary

${ }^{3}$ Department of Pharmacology, Faculty of Pharmacy, University of Debrecen, Debrecen, Hungary

4 Institute of Animal Science, Biotechnology and Nature Conservation, Faculty of Agricultural and Food Sciences and Environmental Management, University of Debrecen, Debrecen, Hungary

${ }^{5}$ Hemostasis, Thrombosis and Vascular Biology Research Group, Hungarian Academy of Sciences, Debrecen, Hungary

Correspondence and reprint request: Arpad Tosaki PhD, DSc

Department of Pharmacology, Faculty of Pharmacy, University of Debrecen

Address: Nagyerdei krt. 98., H-4032 Debrecen, Hungary

Tel/Fax: +36-52-255586 Email: tosaki.arpad@pharm.unideb.hu 


\begin{abstract}
In the present study, we investigated the effects of SCSE on variety of systemic processes which contribute to general health and viability of human subjects. The experiments were conducted according to a double-blind protocol in which six healthy individuals were administered $250 \mathrm{mg} /$ day SCSE for 14 days, while 4 were treated with placebo. Peripheral blood was collected before and after the treatment period. Samples were analyzed for levels of selected cells, enzymes or metabolites. Subjects received SCSE showed increases in the values of mean cell volume, serum transferrin, mean peroxidase index and representation of peripheral blood lymphocytes. On the other hand, decreases were observed in circulating neutrophils, and ferritin levels. Changes observed in the present study do not fit into a clear pattern that might yield additional in-depth understanding of SCSE-mediated alterations in physiologic responses. The most encouraging result of the present study is absence of any indication of toxicity by subjects consuming the extract.
\end{abstract}

Keywords: sour cherry seed extract, heme oxygenase-1, human subjects 


\section{INTRODUCTION}

Ongoing nutritional studies have recently revealed powerful health-promoting properties in components of some plants, which until recently had remained obscure because they were not typically consumed as food by humans. A particularly fascinating example is the seed kernel of sour cherry (Prunus cerasus). The fruit is a major export of several nations, including Hungary; however, the seed is currently considered an agricultural by-product and discarded. Chemical analysis of the seed kernel by authors of the present investigation revealed that the solid flavonoid-rich fraction, comprising approximately 64-68\% of the kernel contains several health-enhancing polyphenolic compunds such as catechins, stillbenes, and anthocyanidins. The remaining $32-36 \%$ is an oil fraction rich in tocopherols and related compounds, including tocotrienols, oleic acid and triglycerides (Bak, Lekli et al. 2010). Results of work with sour cherry seed extract (SCSE) include demonstrations that oral administration of seed kernel meal to animals strongly stabilizes healthy tissue homeostasis and suppresses ischemia-reperfusion (IR) injury by augmenting expression of heme oxygenase-1 (HO-1), a major endogenous cytoprotective enzyme (Szabo, Gallyas et al. 2004, Czompa, Gyongyosi et al. 2014).

Administration of the extract to animals as a feed supplement is observed to prevent and counteract retinal and myocardial IR injury in rats (Szabo, Gallyas et al. 2004, Czompa, Gyongyosi et al. 2014). Powerful cardioprotective effects were noted in hypercholesteremic rabbits fed with seed kernel extract. In these experiments extract-treated animals exhibited significantly improved cardiac function, diminished infarct size and atherosclerotic plaque formation, along with other indicators of resistance to the hypercholesteremic condition by animals receiving sour cherry seed extract (Juhasz, Kertesz et al. 2013). Further evidence for 
potential therapeutic benefit of SCSE components was provided by Mahmoud et al showing that sour cherry seed solid fraction suppressed LPS-mediated expansion of human $\mathrm{T}$ cells activated to express TNF-a to a greater degree in cells taken from human type 2 diabetics, than from cells contributed by healthy subjects (Mahmoud, Al-Awadhi et al. 2013). This is particularly significant since TNF-alpha is a major mediator of insulin resistance and dysregulated inflammation in type 2 diabetes (Hotamisligil, Shargill et al. 1993, Hotamisligil 2006, Hooper and Hooper 2009). The toxicological evaluation of the seed extract, which showed that oral administration at dosages in the range of 200x therapeutic level, did not result in any adverse effects, as evidenced by studies of liver and kidney enzymatic functions and immunohistochemistry (Bak, Czompa et al. 2011).

Protection of rat retinas and rabbit hearts by sour cherry seed kernel components, along with suppression of pro-inflammatory signaling by leukocytes from in human diabetics, all correlated strongly with extract-mediated induction of heme oxygenase- 1 in the experimental models used for these studies (Szabo, Gallyas et al. 2004, Juhasz, Kertesz et al. 2013, Mahmoud, Al-Awadhi et al. 2013) Heme oxygenases are an essential endogenous antioxidant defense, expressed ubiqutously by most tissues in a wide variety of life forms (Haines, Lekli et al. 2012).

The present study evaluates the capacity of SCSE to affect a variety of systemic processes which contribute to general health and viability of human subjects. The SCSE dosage administered to subjects during this initial phase of a comprehensive program of human trials was for safety reasons far below the dosage observed to exert therapeutic effects in animals (Szabo, Gallyas et al. 2004). A major objective in utilization of a subclinical dose 
range was to observe data trends resulting from oral SCSE treatment, without attendant risk (albeit, not expected) from high dose intake of the extract. 


\section{METHODS}

\section{Human subjects}

Participants in the present investigation included 10 healthy volunteers (4 males and 6 females), recruited from the staff of University of Debrecen Health Science Center. Individuals were screened for eligibility according to the following inclusion and exclusion criteria. Inclusion criteria for participation include the following: healthy individuals of both genders in an age range of 18-65 years and in good health, with no major disease. The following individuals were excluded from the present study: (a) persons afflicted at the time of recruitment with ongoing acute or chronic disease; (b) persons with histories of acute or chronic disease severe enough to require hospitalization and/or use of prescription drugs within 3 months of screening for participation in the study; (c) organ transplant recipients; (d) chronic substance abusers; (e) individuals who exhibit positive allergic response to sour cherry seed extract; (f) convalescents of any trauma more recent than 3 months. Informed written consent was obtained from the subjects, and the study has been approved by the Ethics Committee of the University of Debrecen, Hungary. All experiments carried out were in compliance with the Declaration of Helsinki. Both the investigators and participants in this investigation were blinded with respect to whether each oral treatment contained SCSE or placebo.

\section{Test cohorts and treatments}

Subjects were randomized into a test group of 6 subjects, each of whom was administered $250 \mathrm{mg}$ SCSE daily for 14 days; and a control group of 4 subjects sham-treated with placebo (sugar).

\section{Sour cherry seed extract}


Sour cherry seeds were collected by Jonaco, Ltd. (Nyírtass, Hungary) from orchards in the locale of Nyírtass after harvesting and processing the fruit. The seeds were dried, and the kernels removed from the external shell components. The kernels were then macerated into a paste with a consistency suitable for oral administration to humans. This material was encapsulated in gelatin caplets at the bioproduction manufacturing facility belonging to the Central Pharmacy of the University of Debrecen (Debrecen, Nagyerdei str. 98, H-4032, Hungary).

\section{Outcome measurements}

Baseline data was taken from each participant, including: age, body weight and height, body mass index (BMI), mid-abdominal circumference, body fat percentage and skeletal muscle percentage.

Peripheral blood was collected at the baseline, before the first intake of SCSE or placebo, and at the end of the treatment period. Peripheral blood white blood cell count (WBC), red blood cell count (RBC), hemoglobin (Hgb), hematocrit (HCT), mean cell volume (MCV), mean corpuscular hemoglobin $(\mathrm{MCH})$, mean corpuscular hemoglobin concentration (MCHC), red cell distribution width (RDW), platelet cell count (PLT), mean platelet volume (MPV), platelet distribution width (PDW), mean peroxidase index (MPXI), and ratios of lymphocytes, monocytes, neutrophils, eosinophils and basophils were evaluated using an Advia 2120 hematology analyzer (Siemens Medical Solutions Diagnostics Europe Limited, Dublin, Ireland). The Cobas 8000 modular analyzer series (Roche Diagnostics GmbH, Mannheim, Germany) was used to evaluate blood of participants for total bilirubin (tBil), conjugated bilirubin (kBil), high- and low density lipoprotein (HDL \& LDL), triglycerides (TGL), alkaline phosphatase (AP), amylase (AM), lipase (LP), magnesium, c-reactive protein (CRP), iron, transferrin, ferritin, glutamic-oxaloacetic transaminase (GOT), glutamic pyruvic 
transaminase (GPT), and lactate dehydrogenase (LDH). The aforementioned analyses were conducted at the Department of Laboratory Medicine, University of Debrecen. Plasma levels of HO-1 protein were determined at the Food Analytical Laboratory of University of Debrecen by using the StressXpressTM Human HO-1 ELISA Kit (Enzo Life Sciences International, Inc., Plymouth Meeting, Pennsylvania, USA). Briefly, plasma from each participant was incubated in 96-well microtiter plate coated with an antihuman HO-1 antibody, followed by treatment with secondary/detect antibody and related reagents provided with kits. Plasma HO-1 levels were evaluated during the absorbance of the developed kit reagents at $450 \mathrm{~nm}$ in a Biotek ELX 808 Microplate Reader. Results are reported in nanograms (ng) of HO-1 protein per $\mathrm{ml}$ of plasma as median values of each of 4 ELISA outcomes (quadruplicates) within the designated interquartile range (IR) for the Enzo kits used to accomplish these HO-1 assays.

\section{Statistical analyses}

The SPSS ver. 20.0 (SPSS Inc., Chicago, IL, UDA) was used for statistical analysis. To assess the distribution of the data Kolgomorov-Smirnov test was used. In cases of normal distribution, we determined mean \pm standard deviation $(\mathrm{SD})$ values and used two-sample $t$ test for statistical comparison of the experimental data. In cases of distributions different from that of normal, median, minimum and maximum values were calculated, and Mann-Whitney test was used. According to the distribution, Paired samples t test or Wilcoxon test was used to evaluate the changes in distinctive parameters. Differences were considered statistically significant at $\mathrm{p}<0.05$.

\section{RESULTS}




\section{Assessment of peripheral blood tests}

Demographic data and results of biometric measurements are shown in Table 1. As shown in Table 2, relative to control participants not administered SCSE, subjects treated with the extract exhibited small but significant increases in the values of mean cell volume (MCV) $(\mathrm{p}=0.024)$, serum transferrin $(\mathrm{p}=0.035)$, mean peroxidase index $(\mathrm{MPXI})(\mathrm{p}=0.015)$ and representation of peripheral blood lymphocytes $(\mathrm{p}=0.016)$. Table 2 also shows outcome variables significantly decreased in SCSE-treated participants relative to placebo-treated control subjects. These outcomes included decreases observed in circulating neutrophils $(\mathrm{p}=0.019)$, and ferritin $(\mathrm{p}=0.039)$. The significant changes in outcome variables were observed to occur only in the SCSE-treated subjects and not in placebo-treated participants. Ferritin levels of the two patients group could not be statistically compared, since the normal ranges of serum ferritin significantly differ between females and males.

\section{Plasma levels of $\mathrm{HO}-1$ protein}

Time course evaluation of plasma HO-1 levels is shown in Figure 1. Comparison of baseline plasma HO-1 concentration with measurements taken at 1 - and 2 weeks posttreatment demonstrates that SCSE at the dosage provided had no significant effects on systemic HO-1 levels.

\section{DISCUSSION}

The present study, in which SCSE was administered to human volunteers at dosages substantially below the dose range observed to result in clinical effects in animals (Szabo, Gallyas et al. 2004, Bak, Lekli et al. 2010, Juhasz, Kertesz et al. 2013, Czompa, Gyongyosi et al. 2014) was structured to meet three major criteria: First, a panel of 32 hematological, 
serological and blood chemistry assays were selected as basic screening measures for effect of SCSE consumption, that encompassed a broad range of physiological activities known to be affected by disease. Utilization of highly automated testing for such a widely cast net allowed cost-effective assessment of multiple bioparameters that might be altered by the effect of the product. To best focus the present report on major outcomes of highest clinical relevance, only biomarkers that exhibited statistically significant changes as a result of SCSE oral treatment were displayed in the tables. Secondly, since these experiments constituted the first evaluation of orally delivered SCSE in humans, it was important to configure the investigation to avoid toxic effects, while nevertheless producing outcomes useful in predicting applications of the extract in preventive medicine and therapy. Third, although it was expected that use of substantially subclinical dosages of the extract would fail to produce dramatic changes in the parameters measured, the authors nevertheless anticipated that the dose range used would result in data trends expected to become more pronounced at dosages in the therapeutic range.

Outcomes of the present study were considered not suitable as the basis for broad generalizations concerning the biological effects of SCSE, since only a small and heterogeneous group of human subjects participated. It must nevertheless be emphasized that the main purpose of this work was not in-depth characterization of the material, instead, a wide range of physiologic responses were screened for response trends that may indicate potentially toxic response to the product. Although the sample $\mathrm{n}$ for each assay was small, statistically valid $(\mathrm{p}<0.05)$ conclusions regarding SCSE effect on increase or decrease of a particular parameter may be made by comparison of persons receiving extract versus placebo, as shown in Tables 1 and 2 . 
Previous studies have demonstrated that SCSE affects disease-associated hematologic and immune outcomes through increased HO-1 activation. Thus, analysis of the results of the present study focused on likely effects of this enzyme. It is nevertheless important to qualify the significant changes in hematologic and immune parameters by emphasizing that although these are clearly SCSE-associated, there is insufficient data shown here to ascribe these aforementioned effects to SCSE-induced changes in HO-1 activity since the experiment does not control for this variable by observing whether specifically inhibiting HO-1 activity also inhibits the observed response. Moreover, since consumption of the extract failed to result in significantly increased plasma levels of the enzyme, no conclusions can be made based on the present data about the correlation between HO-1 activity and the hematologic and immune effects.

\section{Outcome measures significantly increased following SCSE treatment.}

Significant increases were observed in mean cell volume (MCV), transferrin, Mean peroxidase index (MPXI) and Lymphocyte percentage (LYMP \%) in subjects administered SCSE, with no corresponding significant change in untreated (control) subjects. Possible contributors to these increases are discussed below.

$\mathrm{MCV}$ is a measure of the average red blood cell volume that is reported as part of a standard complete blood count. The normal reference range is typically $80-100$ femtoliters (fL). The significant increase in this variable noted following SCSE treatment may be due to an effect of one or more components of the extract on differentiation of erythroid precursors in the bone marrow in ways which contribute to increase in red blood cell volume.

Transferrins are iron-binding blood glycoproteins that control the level of free iron in biological fluids. Normal reference ranges for transferrin are 2.04-3.60 g/L. Significant increases in serum transferrin observed in SCSE-treated subjects may be related to MCV 
levels based on the capacity of transferrin proteins to facilitate delivery of iron to erythroid progenitors of red blood cells via the transferrin receptor (Macedo and de Sousa 2008). A mechanism for modulation of this process is suggested by results of a recent study demonstrating that sustained induction of HO-1 decreased TR expression, along with several corollary alterations in cellular processing of iron ( $\mathrm{Li}$, Lonn et al. 2012). It is therefore possible that the slight increase in transferrin observed in the present study may be an adaptive response to maintain constant cellular iron intake levels due to decreased TR availability as a result of HO-1 induction due to SCSE intake.

MPXI, which is a measure of neutrophil peroxidase activity, is observed to be decreased in persons afflicted with iron deficiency-associated anemia and increased as a result of iron replacement indicating the contribution of normal peroxidase activity to healthy iron metabolism. The reference range for this parameter is -10 to +10 arbitrary units (Gulley, Bentley et al. 1990). The positive correlation of MPXI with SCSE treatment may be unrelated to induction of HO-1 by the extract since it has been shown that HO-1 suppresses myeloperoxidase activity as a major feature of its capacity to ameliorate inflammatory tissue damage (Lin, Lai et al. 2013). The effect noted may alternatively be a consequence of immunogenicity by plant lectins in the extract (Moreira Rde, Ainouz et al. 1991), an effect that may also account for elevation in lymphocyte representation described below. It is also possible that SCSE-mediated upregulation of transferrin levels and MCV altered iron metabolism in ways that are reflected by increased myeloperoxidase activity and elevated MPXI.

Peripheral blood lymphocytes, which include T, B and natural killer cells (NK cells) act as major mediators of immune activity, coordinating primary and antigen driven immune responses and antitumor functions (Charles A Janeway, Paul Travers et al. 2001). In normal healthy humans, the percentage representation of these cells ranges from $\sim 16-45 \%$ of the total 
white blood cell component. Proliferation of lymphocytes occurring in response to viral or bacterial infections, autoimmunity and cancer is unlikely to account for the very minor increase in this parameter observed in SCSE-treated participants. The increase in percentage of circulating lymphocytes noted in the present study, although significant (0.016) was minor in magnitude and probably not related to induction of heme oxygenase as a result of the SCSE treatment. Indeed, the anti-inflammatory effects of the extract were more likely to have stabilized lymphocyte count than elevated it based on the increased immunogenicity conferred on tissues by inflammatory processes (Charles A Janeway, Paul Travers et al. 2001). Other possible explanations for this observation is that SCSE may contain a meyloperoxidase inducer, possibly a plant lectin, which are strongly immunogenic carbohydrate-binding proteins produced by a wide variety of plants (Moreira Rde, Ainouz et al. 1991). These possibilities are nevertheless speculative and await more detailed studies for resolution.

\section{Outcome measures significantly decreased following SCSE treatment.}

Circulating neutrophil percentage (NEUT \%) and ferritin were observed to significantly decrease in subjects administered SCSE, with no corresponding significant change in untreated (control) subjects. Neutrophils, which are the most frequently occuring white blood cell, serving as a first line of defense against microbial invaders (Witko-Sarsat, Rieu et al. 2000). Once activated, these cells contribute substantially to oxidative stress placed on tissues incidental to their antimicrobial function (Khansari, Shakiba et al. 2009). Reference range in peripheral blood: 35\%-80\%. The observed decrease in peripheral blood representation of these cells with SCSE treatment is consistent with observations that upregulation of endogenous antioxidants, including heme oxygenase, suppresses inflammatory tissue damage through mechanisms that include decreased numbers and activity of neutrophils (Guo and Ward 2007). Ferritin is a widely distributed protein which complexes 
with iron and releases it in response to physiologic cues, serving as a reserve store of iron for an organism (Granier, Langlois d'Estaintot et al. 2003). The level of ferritin in blood and other fluids therefore reflects the quantity of iron available for maintenance of normal homeostatic functions (Theil 1987). Moreover, iron acts as an inducer for ferritin production to maintain levels of the protein sufficient to keep pace with iron storage requirements (Andrews, Arosio et al. 1992). HO-1 metabolizes heme and releases ferrous iron $\left(\mathrm{Fe}^{2+}\right)$, which is an inducer of ferritin (Grochot-Przeczek, Dulak et al. 2012). The significant decrease in levels of this metabolite observed in response to SCSE treatment may be a consequence of HO-1 induction by the extract, which, despite the non-significant changes in its activity, may have occurred at levels sufficient to account for the small decrease in ferritin levels. Outcomes of the present study demonstrate SCSE treatment-associated changes in bioparameters that might reflect protective properties of the extract in cells or animals subjected to pathological stress as part of the experimental design. Indeed, this flavonoid-rich extract has demonstrated powerful protective properties in previous studies. It should nevertheless be emphasized that the subjects of this study were healthy and this investigation was undertaken primarily to assess indications of biomarker expression trends that with larger sample sizes could be indicators of toxicity.

\section{Conclusions}

The significantly altered parameters observed in blood of SCSE-treated subjects but not in untreated controls may or may not be related to induction of HO-1 expression by the extract. Sufficient data to support or refute such a possibility has not been produced in this study and the lack of significant HO-1 induction by the SCSE dosage administered, makes any correlations of this kind highly speculative. Moreover, the changes observed in the present study do not fit into a clear pattern that might yield additional in-depth understanding 
of SCSE-mediated alterations in physiologic responses, although some of the results suggest that the extract is affecting iron metabolism at some - which in turn might be related to HO-1 activity based on the known properties of the enzyme ( $\mathrm{Li}$, Lonn et al. 2012). The most encouraging result of the present study is absence of any indication of toxicity by subjects consuming the extract. Gross toxic effects were not expected based on animal studies conducted by the authors demonstrating non-toxicity of the extracts at dosages in the range of 200x therapeutic (Bak, Czompa et al. 2011). It is very encouraging to note that subjects administered the extract exhibited no increases in the activity of glutamic-oxaloacetic transaminase (GOT) and glutamic pyruvic transaminase (GPT) which are liver function biomarkers used as indicators of drug toxicity (Atasoy, Erdogan et al. 2007), nor were any significant changes in the activity of plasma lactate dehydrogenase (LDH), which is an indicator of disrupted tissue homeostasis used for evaluation of herbal preparation safety profiles (Liu, Liu et al. 2013). Finally, a major objective of the efforts to determine the full scope of healthcare, cosmetic and dietary uses for SCSE is establishment of a solid safety profile as a necessary precondition for its human use. Previous studies have yielded very encouraging results. For example, no known toxicants were identified in chemical analysis of the seed kernel components (Bak, Lekli et al. 2010), moreover sustained high dose oral administration of SCSE failed to result in liver or kidney toxicity (Bak, Czompa et al. 2011). Ongoing efforts to gain an in-depth understanding of the effects of this substance, include experiments described in the present report; and a phase I clinical trial of SCSE delivered transdermally to osteoarthritic patients. This treatment resulted in significant systemic amelioration of multiple disease parameters (including joint pain) and augmented protective $\mathrm{T}$ lymphocyte activity, with no associated toxicity (Mahmoud, Haines et al. 2014). These outcomes and ongoing results of work-in-progress suggest that SCSE will be safe and effective for a wide range of human applications. 


\section{ACKNOWLEGEMENTS}

This study was supported by grants from OTKA K-104017 (A.T.) PD-111794 (I.L.), the Janos Bolyai Research Scholarship (I.L.), the Lajos Szodoray Scholarship (I. L.), Grant of UD (I.B., I.L.), TÁMOP- 4.2.2.A-11/1/KONV-2012-0045 (A.T.) projects co-financed by the European Union and the European Social Fund and MTA-DE Vascular Biology, Thrombosis and Hemostasis Research Group by the Hungarian Academy of Sciences (MTA-DE Vasc. Biol. 11003). A.Czo., I.L., A.T. assisted in fundamental research in the frame of TÁMOP 4.2.4. A/2-11-1-2012-0001 National Excellence Program - Elaborating and operating an inland student and researcher personal support system, was realized with personal support. The project was subsidized by the European Union and co-financed by the European Social Fund."The authors report no conflicts of interest. 


\section{REFERENCES}

Andrews, S. C., P. Arosio, W. Bottke, J. F. Briat, M. von Darl, P. M. Harrison, J. P. Laulhere, S. Levi, S. Lobreaux and S. J. Yewdall (1992). "Structure, function, and evolution of ferritins." J Inorg Biochem 47(3-4): 161-174.

Atasoy, N., A. Erdogan, I. Yalug, U. Ozturk, N. Konuk, L. Atik and Y. Ustundag (2007). "A review of liver function tests during treatment with atypical antipsychotic drugs: a chart review study." Prog Neuropsychopharmacol Biol Psychiatry 31(6): 1255-1260.

Bak, I., A. Czompa, E. Csepanyi, B. Juhasz, H. Kalantari, K. Najm, N. Aghel, B. Varga, D. D. Haines and A. Tosaki (2011). "Evaluation of systemic and dermal toxicity and dermal photoprotection by sour cherry kernels." Phytother Res 25(11): 1714-1720.

Bak, I., I. Lekli, B. Juhasz, E. Varga, B. Varga, R. Gesztelyi, L. Szendrei and A. Tosaki (2010). "Isolation and analysis of bioactive constituents of sour cherry (Prunus cerasus) seed kernel: an emerging functional food." J Med Food 13(4): 905-910.

Charles A Janeway, J., Paul Travers, Mark Walport and M. J. Shlomchik (2001). Immunobiology, 5th edition. New York; USA, Garland Science.

Czompa, A., A. Gyongyosi, A. Czegledi, E. Csepanyi, I. Bak, D. D. Haines, A. Tosaki and I. Lekli (2014). "Cardioprotection afforded by sour cherry seed kernel: the role of heme oxygenase-1." J Cardiovasc Pharmacol.

Granier, T., B. Langlois d'Estaintot, B. Gallois, J. M. Chevalier, G. Precigoux, P. Santambrogio and P. Arosio (2003). "Structural description of the active sites of mouse L-chain ferritin at 1.2 A resolution." J Biol Inorg Chem 8(1-2): 105-111.

Grochot-Przeczek, A., J. Dulak and A. Jozkowicz (2012). "Haem oxygenase-1: noncanonical roles in physiology and pathology." Clin Sci (Lond) 122(3): 93-103.

Gulley, M. L., S. A. Bentley and D. W. Ross (1990). "Neutrophil myeloperoxidase measurement uncovers masked megaloblastic anemia." Blood 76(5): 1004-1007. 
Guo, R. F. and P. A. Ward (2007). "Role of oxidants in lung injury during sepsis." Antioxid Redox Signal 9(11): 1991-2002.

Haines, D. D., I. Lekli, P. Teissier, I. Bak and A. Tosaki (2012). "Role of haeme oxygenase-1 in resolution of oxidative stress-related pathologies: focus on cardiovascular, lung, neurological and kidney disorders." Acta Physiol (Oxf) 204(4): 487-501.

Hooper, P. L. and P. L. Hooper (2009). "Inflammation, heat shock proteins, and type 2 diabetes." Cell Stress Chaperones 14(2): 113-115.

Hotamisligil, G. S. (2006). "Inflammation and metabolic disorders." Nature 444(7121): 860-867.

Hotamisligil, G. S., N. S. Shargill and B. M. Spiegelman (1993). "Adipose expression of tumor necrosis factor-alpha: direct role in obesity-linked insulin resistance." Science 259(5091): 87-91.

Juhasz, B., A. Kertesz, J. Balla, G. Balla, Z. Szabo, M. Bombicz, D. Priksz, R. Gesztelyi, B. Varga, D. D. Haines and A. Tosaki (2013). "Cardioprotective Effects of Sour Cherry Seed Extract (SCSE) on the Hypercholesterolemic Rabbit Heart." Curr Pharm Des.

Khansari, N., Y. Shakiba and M. Mahmoudi (2009). "Chronic inflammation and oxidative stress as a major cause of age-related diseases and cancer." Recent Pat Inflamm Allergy Drug Discov 3(1): 73-80.

Li, C., M. E. Lonn, X. Xu, G. J. Maghzal, D. M. Frazer, S. R. Thomas, B. Halliwell, D. R. Richardson, G. J. Anderson and R. Stocker (2012). "Sustained expression of heme oxygenase-1 alters iron homeostasis in nonerythroid cells." Free Radic Biol Med 53(2): 366-374. 
Lin, Y. C., Y. S. Lai and T. C. Chou (2013). "The protective effect of alpha-lipoic Acid in lipopolysaccharide-induced acute lung injury is mediated by heme oxygenase-1." Evid Based Complement Alternat Med 2013: 590363.

Liu, Z. L., Z. J. Liu, J. P. Liu and J. S. Kwong (2013). "Herbal medicines for viral myocarditis." Cochrane Database Syst Rev 8: CD003711.

Macedo, M. F. and M. de Sousa (2008). "Transferrin and the transferrin receptor: of magic bullets and other concerns." Inflamm Allergy Drug Targets 7(1): 41-52.

Mahmoud, F., D. Haines, R. Al-Awadhi, A. A. Dashti, A. Al-Awadhi, B. Ibrahim, B. AlZayer, B. Juhasz and A. Tosaki (2014). "Sour cherry (Prunus cerasus) seed extract increases heme oxygenase-1 expression and decreases proinflammatory signaling in peripheral blood human leukocytes from rheumatoid arthritis patients." Int Immunopharmacol 20(1): 188-196.

Mahmoud, F. F., R. Al-Awadhi, D. D. Haines, A. Dashti, H. Dashti, E. Al-Ozairi, I. Bak and A. Tosaki (2013). "Sour cherry seed kernel extract increases heme oxygenase-1 expression and decreases representation of CD3+ TNF-alpha+ and CD3+IL-8+ subpopulations in peripheral blood leukocyte cultures from type 2 diabetes patients." Phytother Res 27(5): 767-774.

Moreira Rde, A., I. L. Ainouz, J. T. De Oliveira and B. S. Cavada (1991). "Plant lectins, chemical and biological aspects." Mem Inst Oswaldo Cruz 86 Suppl 2: 211-218.

Szabo, M. E., E. Gallyas, I. Bak, A. Rakotovao, F. Boucher, J. de Leiris, N. Nagy, E. Varga and A. Tosaki (2004). "Heme oxygenase-1-related carbon monoxide and flavonoids in ischemic/reperfused rat retina." Invest Ophthalmol Vis Sci 45(10): 37273732.

Theil, E. C. (1987). "Ferritin: structure, gene regulation, and cellular function in animals, plants, and microorganisms." Annu Rev Biochem 56: 289-315. 
Witko-Sarsat, V., P. Rieu, B. Descamps-Latscha, P. Lesavre and L. HalbwachsMecarelli (2000). "Neutrophils: molecules, functions and pathophysiological aspects." Lab Invest 80(5): 617-653.

\section{TABLES}

\begin{tabular}{|l|l|l|l|}
\hline Bioparameter & $\begin{array}{l}\text { Test subjects to be } \\
\text { administered SCSE } \\
(\mathbf{n}=6)\end{array}$ & $\begin{array}{l}\text { Control subjects } \\
\text { receiving placebo } \\
(\mathbf{n}=4)\end{array}$ & \\
\hline Age (years) & $38.17 \pm 2.48$ & $45.50 \pm 6.81$ & $\mathrm{p}<0.05$ \\
\hline Female/male & $2 / 4$ & $4 / 0$ & \\
\hline Body weight (kg) & $86.50 \pm 12.34$ & $87.50 \pm 5.00$ & n.s. \\
\hline Height (cm) & $174.67 \pm 5.79$ & $163.25 \pm 5.12$ & $\mathrm{p}<0.05$ \\
\hline Body mass index (BMI) & $28.92 \pm 5.454$ & $25.42 \pm 6.54$ & n.s. \\
\hline Belly circumference (cm) & $100.17 \pm 12.79$ & $87.00 \pm 12.62$ & n.s. \\
\hline Body fat (percentage by & $31.50 \pm 5.36$ & $37.55 \pm 10.26$ & n.s. \\
\hline weight) & & & \\
\hline Skeletal muscle (percentage & $32.53 \pm 4.86$ & $25.68 \pm 3.51$ & $\mathrm{p}<0.05$ \\
\hline by weight) & & & \\
\hline & & & \\
\hline
\end{tabular}

Table 1. Demographical data and biometric measurements of test and control participants. Each bioparameter shown below was assessed at outset of study, prior to administration of first SCSE or placebo treatment. 


\begin{tabular}{|c|c|c|c|c|}
\hline Outcome variable & & $\begin{array}{l}\text { Test subjects SCSE } \\
\text { treated with oral } \\
\text { SCSE }(n=6)\end{array}$ & $\begin{array}{l}\text { Control } \\
\text { subjects } \\
\text { receiving } \\
\text { placebo }(n=4)\end{array}$ & $p$ value \\
\hline \multirow{3}{*}{ Mean cell volume (MCV, fL) } & before & $90.00 \pm 3.54$ & $91.30 \pm 2.44$ & 0.543 \\
\hline & after & $90.87 \pm 3.68$ & $91.70 \pm 3.49$ & 0.730 \\
\hline & $\mathrm{p}$ value & 0.024 & 0.675 & \\
\hline \multirow{3}{*}{ Transferrin $(g / L)$} & before & $2.70 \pm 0.27$ & $2.73 \pm 0.22$ & 0.871 \\
\hline & after & $2.84 \pm 0.22$ & $2.92 \pm 0.39$ & 0.697 \\
\hline & $\mathrm{p}$ value & 0.035 & 0.188 & \\
\hline \multirow{3}{*}{$\begin{array}{l}\text { Mean peroxidase index } \\
\text { (MPXI, arbitrary units) }\end{array}$} & before & $-9.25 \pm 15.81$ & $-4.10 \pm 6.21$ & 0.558 \\
\hline & after & $-7.87 \pm 15.49$ & $-4.40 \pm 6.79$ & 0.689 \\
\hline & $\mathrm{p}$ value & 0.015 & 0.682 & \\
\hline \multirow{3}{*}{$\begin{array}{l}\text { Peripheral blood lymphocytes } \\
(\text { LYMPH, \%) }\end{array}$} & before & $24.33 \pm 5.66$ & $28.55 \pm 8.89$ & 0.381 \\
\hline & after & $29.08 \pm 5.08$ & $29.40 \pm 12.01$ & 0.955 \\
\hline & $\mathrm{p}$ value & 0.016 & 0.734 & \\
\hline \multirow{3}{*}{$\begin{array}{l}\text { Peripheral blood neutrophils } \\
(\text { NEUT, \%) }\end{array}$} & before & $65.67 \pm 7.30$ & $60.78 \pm 8.57$ & 0.360 \\
\hline & after & $59.17 \pm 6.92$ & $61.22 \pm 12.37$ & 0.742 \\
\hline & $\mathrm{p}$ value & 0.019 & 0.866 & \\
\hline \multirow{3}{*}{ Ferritin (mg/L) } & before & $176.05 \pm 123.99$ & $38.95 \pm 23.45$ & N/A \\
\hline & after & $154.73 \pm 111.36$ & $35.25 \pm 23.95$ & N/A \\
\hline & $\mathrm{p}$ value & 0.039 & 0.238 & \\
\hline
\end{tabular}

Table 2. Outcome variables exhibiting significant changes following oral administration of sour cherry extract. Relative to control participants not administered SCSE, subjects 
treated with the extract exhibited small but significant increases in the values of MCV, transferrin, MPXI and representation of peripheral blood lymphocytes. Relative to control participants not administered SCSE, subjects treated with the extract exhibited small but significant decreases in the values of circulating neutrophils and ferritin. These changes in outcome variables were observed to occur only in the SCSE-treated subjects and not in placebo-treated participants. N/A: not applicable.

\section{FIGURE LEGENDS}

Figure 1. Plasma heme oxygenase-1 (HO-1) levels. Plasma from SCSE-treated test subjects $(n=6)$ and untreated control participants $(n=4)$ was evaluated for concentration of HO-1 protein using the StressXpressTM Human HO-1 ELISA Kit before SCSE treatment (baseline), 1 week and 2 weeks following administration of extract. Bars show the mean and the standard deviation (SD). No significant effects noted. 